Es ist eine moderne Grammatik, die alles auch in strukturalistischer Optik darzustellen vermag, womit sie die Anwendung der Sprache in computerunterstützten Situationen ermöglicht.

Stojan Bračič

\title{
Nina Janich: WERBESPRACHE. EIN ARBEITSBUCH. Gunter Narr Verlag, Tübingen, 1999
}

Gegenstand des zu besprechenden Buches von Nina Janich ist das Phänomen Werbesprache, „das nicht nur als Kulisse ... überall präsent ist ..., sondern das auch immer mehr Kult- und Kunststatus und damit ausdrückliche Aufmerksamkeit erhält" (7). Wer sich heute mit der Sprache beruflich beschäftigt und sie analysiert, kommt um die Werbesprache nicht herum: fast alle modernen Tendenzen der Sprachentwicklung lassen sich daran nachweisen, und wenn Werbesprache aus inflationären Grunden nicht gerade als Hauptkorpus für Analysen ausgesucht wird, so kann man kaum der Versuchung widerstehen, für gewisse Hypothesen mindestens sporadisch doch auch werbesprachliches Belegmaterial heranzuziehen. Bislang hat es kein Werk gegeben, das sich so systematisch und umfassend mit der Werbesprache beschäftigt wie das Arbeitsbuch von Nina Janich. Es bringt einleitend einen theoretischen Rahmen über „Markt und Kommunikation“, präsentiert danach die Bausteine der Werbung, untersucht Werbetexte unter verschiedenen sprachwissenschaftlichen Forschungsaspekten, um mit methodischen Tipps zu Analysen und einigen Überlegungen zur Diachronie und zu den Fragen der Interkulturalität in Werbeuntersuchungen zu schließen. Das Buch von Nina Janich ist, wie bereits oben ausgeführt, als ein Arbeitsbuch konzipiert, das bedeutet, das zu jedem Kapitel Fragen formuliert sind, die zu vertiefender Auseinandersetzung mit der Materie anregen sollen. Lösungsvorschläge zu den Aufgaben befinden sich im Anhang.

In dem einleitenden, mehr theoretischen Kapitel (11 ff.) werden die Geschichte der Sprachwissenschaft rund um die Werbesprache und deren Desiderate diskutiert. Im Anschluss daran wird Werbung definiert (16) und einige grundlegende Elemente dieses Phänomens (Werbeobjekte, Werbeziele, Werbewirkung, Zielgruppenbestimmung, Werbemittel, Werbeträger) beleuchtet. Vor dem Hintergrund verschiedener Kommunikatiosmodelle wird Werbesprache als „eine inszenierte Form von Kommunikation“ präsentiert. Das tiefgreifende Dilemma zwischen Manipulation und/oder Information wird aufgeworfen.

Im sog. „Mikrokosmos Anzeige“ (40) werden die wesentlichen Bausteine der Werbung dargestellt: Schlagzeile, Fließtext, Slogan, Logo, Produktname, besondere Formen 
von Texten (wie z. B. Inserts/Einklinker und Claims/Abbinder), unterschieden wird zwischen primären, sekundären und tertiären Texten, Bildelemente als semiotischer Code in der Werbung und deren funktionale Klassifizierung werden unter die Lupe genommen (58). Mit anschaulichen Belegen werden Begriffe wie Symptom/Index, Ikon und Symbol voneinander abgehoben (60) und auf ihre Überschneidungsvarianten hin geprüft. Ein Verdienst des Buches ist auch darin zu sehen, dass es sich nicht nur auf schriftliche Werbetexte beschränkt, sondern immer wieder Parallelen zu den Fernsehspots als mündliche Variante der Werbehandlung zieht. (64)

Nach der Absteckung des pragmatischen Rahmens (Absicht-Inhalt-Form, Textfunktion, Textthema (72) und Texthandlung (68ff.)), der Aufschlüsselung der persuasiven Funktionen von Sprache (80) und einer eingehenden Erläuterung der Argumentation als wesentliches Kommunikationsverfahren in der Werbekommunikation (82 ff.), wird auf verschiedenen Ebenen die Analyse einzelner sprachlicher Mittel und ihrer Funktionen in Werbetexten unternommen: Lexik (besonders auch Fremdsprachiges, Phraseologie und Wortbildung), Syntax (besonders Satzarten und Satformen in verschiedenen Segmenten der Werbetexte); das Unterkapitel Textgrammatik bringt eine interessante Klassifikation der textkohäsiven Mittel, über die man freilich diskutieren könnte. Der Autorin muss man jedenfals zugeben, dass ihr Vorschlag plausibel und geeignet ist, um praktikable textgrammatische Analysen von Werbetexten durchführen zu können. Nach der Erläuterung der Begriffe Kohäsion und Kohärenz (126) setzt sich die Autorin mit 4 Typen der Vertextungsmittel auseinander: Wiederaufnahme/Rekurrenz, Deixis, Konnexion und Isotopie. Der Vorschlag der Autorin, bei der Rekurrenz mit Begriffen Bezugsausdruck und Verweisausdruck zu operieren und zwischen expliziter und impliziter Wiederaufnahme zu unterscheiden, ist überzeugend und mit gut ausgesuchten Belegen untermauert. Dass Isotopie ein problematisches Kapitel sein kann, wird auch von Nina Janich angedeutet. In dem mit Blitzsymbol abgehobenen Absatz wird mit Recht problematisiert, dass die Isotopie manchmal von der Wiederaufnahme (eine Art der Kontiguität) schwer abzugrenzen ist. (129 uu.) Auch wird angesprochen, dass bei der Isotopie keine Referenzidentität herrschen muss, wobei man der Autorin beipflichten kann - gegenüber einigen anderen Auffassungen (Heinemann/Viehweger), die bei der Isotopie Referenzidentität voraussetzen. Im Grunde genommen geht es dabei aber um eine terminologische Überlappung. Es gibt Ketten von Ausdrücken, die Textrekurrenz herstellen, und zwar auf Grund einer Koreferenzbeziehung oder einer lediglich teilweisen Überschneidung vermittels gemeinsamer Semrekurrenz. Das erste Phänomen kann Koreferenzkette oder Isotopie heißen, das zweite aber Isotopie oder thematische Reihe. Letztendlich kommt es darauf an, dass Phänomene klar präsentiert und voneinander abgegrenzt werden, die Terminologisierung ist dabei zwetirangig. Davon überzeugt auch Nina Janich in ihrem Werk.

Im Unterkapitel „Rhetorik der Werbung" geht es im Grunde um die stilistischen Ausdrucksmittel i.e.S.: der Reihe nach werden in Janichs Werk darunter folgende Fragen behandelt: Textaufbau (das Exordium, die Narration, die Peroratio), rhetorische 
Figuren (Positionsfiguren, Wiederholungsfiguren, Erweiterungsfiguren, Appellfiguren, Tropen) und Sprachspiele (Wortspiele, Kontextspiele, Referenzspiele). Die eingehend und unter verschiedenen Aspekten behandelte Problematik der Sprachspiele ergänzt in mancher Hinsicht die sehr gründliche und umfangreiche Monographie „Sprache und Spiel" von Karl Sornig (Graz, 1993), die aber die Autorin in ihrer Literaturliste nicht anführt. Ein weiteres Unterkapitel beschäftigt sich mit der Funktion von drei Varietäten, die in der Werbesprache Verwendung finden, und zwar der Fachsprache (z. B. Pseudofachsprachlickeit als Merkmal der künstlichen Inszeniertheit), der Jugendsprache (Experimentierung) und des Dialekts (auch Umgangssprache) (163). Dabei wird auf Besonderheiten in Fernsehspots hingewiesen, wo ja bekanntlich hauptsächlich die gesprochene Variante der Werbesprache praktiziert wird.

Sehr wichtig ist in der Werbung die Intertextualität. Ohne Intertextualität in verschiedenen Erscheinungsformen gibt es keinen Werbetext. Mit Recht widmet daher Nina Janich diesem Aspekt der Werbetätigkeit ein relativ umfangreiches Unterkapitel (4.4.3.). Intertextualität wird eingangs als sprachwissenschaftliches Phänomen erläutert, an verschiedenen Belegen aus der Werbung werden dabei verschiedene Typen und Untertypen der Übernahme aus Referenztexten in Phänotexte demonstriert.

Auch der Interpunktion und der Typographie wird im Übungsbuch ein entsprechend umfangreiches Unterkapitel gewidmet. Im Unterkapitel „Text und Bild“ (184) werden verschiedene Beziehungen zwischen Text (,verbale Aussage") und dem ihm komplementären Element Bild (,visuelle Umsetzung“) durchdiskutiert (186 ff.).

Dem Vorschlag eines ganzheitlichen Analysemodells (195) folgen ein illustrativer diachroner Einblick in die Geschichte der werbesprachlichen Forschung und die Überlegungen über die interkulturellen Aspekte der Werbetätigkeit (eine kontrastive und sprachkritische Perspektive).

Das vorliegende Werk ist verständlich geschrieben und enhält viel anschauliches empirisches Material. Es bringt eine komplexe Analyse des Phänomens Werbesprache. Symbole wie z.B. Blitz (Methodische Probleme), Glühbirne (Forschungsanregungen und Desiderate) sorgen dafür, dass man schwierigere Einzelheiten überspringen kann, ohne dass die Verständlichkeit der Perzeption darunter leidet. Kommentierte Literaturtipps sind laufend angeführt und erleichtern bei Bedarf die Wahl der weiterführenden Literatur zu den einzelnen Fragen. Bei der Beschäftigung mit den Aufgaben zu den einzelnen Problemstellungen kann man testen, ob der Stoff sitzt, und die angehängten Lösungsvorschläge sind dabei ein nützliches Feedback. Alle Ebenen des Sprachsystems werden an Werbetexten behandelt und mit den Erkenntnissen der Sprachpragmatik konfrontiert. Das Arbeitsbuch von Nina Janich ist somit nebenbei auch ein kleines Repetitorium einiger wesentlicher Kapitel des sprachwissenschftlichen Wissens.

Inzwischen ist eine zweite Auflage erschienen.

Stojan Bračič 